\title{
The Assimilation of $\gamma$-Butyrolactone in Agrobacterium tumefaciens C58 Interferes with the Accumulation of the N-Acyl-Homoserine Lactone Signal
}

\author{
Aurélien Carlier, Romain Chevrot, Yves Dessaux, and Denis Faure \\ Institut des Sciences du Végétal, UPR2355, Centre National de la Recherche Scientifique, Avenue de la Terrasse, 91198 \\ Gif-sur-Yvette, France
}

Submitted 22 January 2004. Accepted 19 April 2004.

\begin{abstract}
Agrobacterium tumefaciens $\mathrm{C58}$ communicates using $\mathrm{N}$ acyl-homoserine lactones (acyl-HSL) and contains two lactonase-encoding genes, $a t t M$ and $a i i B$, the products of which are capable of inactivating the acyl-HSL signal. In A. tumefaciens A6, the expression of the attKLM operon is controlled by the transcriptional repressor encoded by an adjacent gene, attJ. An attJ::Tn5 mutant does not accumulate acyl-HSL because of the constitutive expression of the lactonase AttM, the activity of which inactivates acyl-HSL. In this work, the attKLM operon of A. tumefaciens $\mathrm{C} 58$ was shown to be involved in an assimilative pathway of $\gamma$-butyrolactone (GBL), $\gamma$-hydroxybutyrate (GHB), and succinate semialdehyde (SSA), in which AttM and AttL are key enzymes for GBL and GHB assimilation. The expression of the attKLM promoter was activated in the presence of GBL, GHB, and SSA. Under these conditions, A. tumefaciens C58 did not accumulate the acyl-HSL that it naturally synthesizes, and also became able to inactivate exogenous acyl-HSL signals. Therefore, in $A$. tumefaciens $\mathbf{C 5 8}$, the assimilative pathway of $\gamma$-butyrolactone interferes with the acyl-HSL signaling.
\end{abstract}

Additional keywords: At plasmid, $N$-3-oxo-octanoyl-L-homoserine lactone, quorum sensing, Ti plasmid.

$\mathrm{N}$-acyl-homoserine lactones (acyl-HSLs) are diffusible pheromones that are produced by many gram-negative bacteria. Beyond a threshold concentration, these signal molecules trigger the expression of target genes via an interaction with specific transcriptional regulators (Fuqua and Greenberg 2002). Numerous bacterial functions are acyl-HSL dependent, including the expression and horizontal transfer of genes encoding virulence factors in plant and animal pathogens (Whitehead et al. 2001). This cell-to-cell communication system that links cell density to gene regulation is termed quorum sensing (QS) (Fuqua et al. 1994).

The search for QS quenchers (i.e., chemical, biochemical, and biological factors disrupting the acyl-HSL-mediated communication) constitutes an original approach to develop novel, antibiotic-free therapies and treatments against pathogenic bacteria in medicine and agronomy (Camara et al. 2002; Zhang 2002). Several QS quenchers have been identified.

Corresponding author: D Faure; Telephone: (33) 1698235 77; Fax: (33) 1698236 95; E-mail: faure@isv.cnrs-gif.fr
They encompass chemical compounds targeting the acyl-HSL synthesis and the turnover of acyl-HSL receptors (Hentzer et al. 2003; Hoang and Schweizer 1999; Manefield et al. 2002), as well as enzymes cleaving acyl-HSL molecules, such as lactonases and acylases (Dong et al. 2000; Lin et al. 2003), and acyl-HSL-degrading bacteria (Dong et al. 2000; Leadbetter and Greenberg 2000; Lin et al. 2003; Uroz et al. 2003). Transgenic plants that express one of these bacterial lactonases were constructed and appeared to be more resistant to QS pathogens than their wild-type parents (Dong et al. 2001).

The above-mentioned lactonases are zinc-hydrolases belonging to the AiiA family, named according to the enzyme AiiA from Bacillus sp. strain 240B1 (Dong et al. 2000). AiiA analogs were discovered in several acyl-HSL-degrading bacterial species (Dong et al. 2002; Park et al. 2003), and in a single acyl-HSL-producing species, the phytopathogen Agrobacterium tumefaciens (Carlier et al. 2003; Zhang et al. 2002). In the octopine-type A. tumefaciens strain A6, the AttM lactonase is encoded by the At plasmid (Zhang et al. 2002). In A. tumefaciens C58, two AiiA lactonases, AttM and AiiB, were identified (Carlier et al. 2003). They are encoded by genes located on the At and nopaline-type Ti plasmids, respectively.

In A. tumefaciens A6, the lactonase-encoding gene attM is part of the attKLM operon that is controlled by the transcriptional repressor AttJ. Upon Tn5 inactivation of attJ, the attKLM operon was constitutively expressed and the corresponding $A$. tumefaciens mutant did not accumulate $N$-3-oxooctanoyl-L-homoserine lactone (oxo-C8HSL), suggesting that the lactonase AttM may be implicated in the modulation of the oxo-C8HSL signal (Zhang et al. 2002). In this work, A. tumefaciens strain C58 was chosen as a model to investigate additional functions of the attKLM operon. This operon encodes an assimilative pathway of $\gamma$-butyrolactone (GBL). Several chemicals that activate its transcription were identified, and novel physiological conditions, in which interference between the expression of attKLM and the accumulation of acyl-HSLs occurred, were defined.

\section{RESULTS}

The genes attM and att $L$ encode key proteins involved in GBL assimilation.

The nucleotide sequence of the attJKLM genes in A. tumefaciens A6 is identical to that in A. tumefaciens C58. These attKLM genes are clustered in an operon (Fig. 1) that is, to date, unique among the sequenced bacterial genomes. The 
attM gene encodes a lactonase that opens the $\gamma$-butyrolactone ring of acyl-HSL, and belongs to the zinc-hydrolase family (Carlier et al. 2003; Zhang et al. 2002). In silico analysis of the deduced amino acid sequences of the two other genes revealed high identities (up to $71 \%$ ) between AttK and several NAD-succinate semialdehyde (SSA) dehydrogenases, and between AttL and several NAD alcohol dehydrogenases (up to $49 \%$ ). The three enzymes (AttK, AttL, and AttM) encoded by this operon may participate in a degradative pathway of GBL, as suggested by the comparison with the catabolic pathway of GBL known in animals (Mason and Kerns 2003). This eukaryotic pathway involves three enzymatic activities: i) a lactonase that converts GBL into $\gamma$-hydroxybutyrate (GHB); ii) a NAD-alcohol dehydrogenase that oxidizes GHB into SSA; and iii) an NAD-SSA dehydrogenase, oxidizing SSA into succinic acid (SA), a compound of the triboxylic acid (TCA) cycle (Figure 2 shows a proposition of such a pathway in A. tumefaciens C58). The characterization of the two first enzymatic steps is crucial to identify a degradation pathway of GBL. To experimentally verify the involvement of AttL and AttM in such a pathway, these two proteins were individually, or simultaneously, expressed in Escherichia coli, a species that naturally assimilates SA and SSA. Although the expression of $a t t L$ was sufficient to convert $E$. coli into an efficient GHB-assimilative bacterium, att $L$ and $a t t M$ were required simultaneously for the growth of $E$. coli on GBL (Table 1). The expression of AttL, however, had no effect on the acyl-HSL signal (Table 1).

\section{The attKLM operon is required}

\section{for the growth of A. tumefaciens on GBL.}

Degradation assays revealed that $A$. tumefaciens C58 did not assimilate a large spectrum of substituted $\gamma$-butyrolactones: $\gamma$ valerolactone, homoserine lactone, and C6HSL cannot be used as sole carbon source. However, strain C58 was able to grow on GBL as sole carbone source, while its $\triangle($ attJKLM) derivative C101 did not. This inability to grown on GBL also was observed when additional copies of the attJ gene encoding the attKLM-transcriptional repressor were introduced into $A$. $t u$ mefaciens C58 (Fig. 3A). The implication of these pAtencoded genes in the assimilation of GBL was in agreement with the phenotypes of strains C58.C2 and C58.00, both lacking pAt, which also did not grow on GBL. In contrast, strain C58.C1, cured of pTi but still harboring pAt, grew on GBL. The mutant $\mathrm{C} 101$ also was affected in the assimilation of GHB and SSA, while no difference was observed between strains

\section{A. tumefaciens $\mathrm{C} 58$}

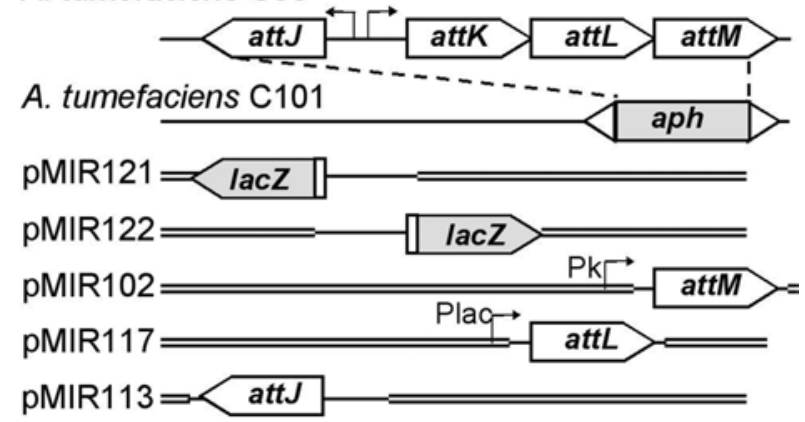

Fig. 1. Schematic representation of the attJKLM region of Agrobacterium tumefaciens $\mathrm{C} 58$ and relevant plasmids used in this study. A. tumefaciens $\mathrm{C} 101$ is an A. tumefaciens C58 derivative in which the attJKLM genes were replaced by aph gene conferring kanamycin resistance. The double lines represent the DNA of cloning vectors, while single lines and open boxes represent pAt-DNA from A. tumefaciens C58. The genes are not drawn to scale relative to each other. $\mathrm{Pk}$ and $\mathrm{Plac}$ are strong bacterial promoters.
C58 and C101 during their growth on SA (Fig. 3B). Based on all these results, the operon attKLM is proposed to be involved in an assimilative pathway of GBL.

\section{GBL, GHB, and SSA stimulate the transcription of attKLM.}

The low copy-number plasmids pMIR121 and pMIR122, harboring the transcriptional fusions attJ::lacZ and attK::lacZ, were introduced into A. tumefaciens $\mathrm{C} 58$ by electroporation. The addition of GBL to bacterial cultures strongly increased the expression of the fusion attK::lacZ, while an equivalent supplementation with mannitol had no effect. In contrast, the expression of the attJ::lacZ fusion was only slightly affected by GBL supplementation (Fig. 4A). The other lactones, such as $\gamma$-valerolactone and homoserine lactone, did not induce the expression of the attKLM operon (data not shown), but the two metabolic intermediates of the GBL assimilative pathway, GHB and SSA, were efficient inducers (Fig. 4B).

\section{Interferences between the expression of attKLM and the acyl-HSL signals.}

When the attKLM operon was not induced (e.g. in the presence of mannitol as sole carbon source), the expression of attK::lacZ transcriptional fusion barely was affected by the addition of C6HSL and oxo-C8HSL, and the concentrations of oxo-C8HSL are equivalent in the cultures of $A$. tumefaciens $\mathrm{C} 58$ and its derivative $\mathrm{C} 101$ (Fig. 5). These results suggested that, in the absence of attKLM inducers in ABmannitol medium, the expression of the attKLM operon is not induced by and does not affect the acyl-HSL concentra-
Inactivation
of acyl-HSL signal

\section{Assimilative pathway for GBL}

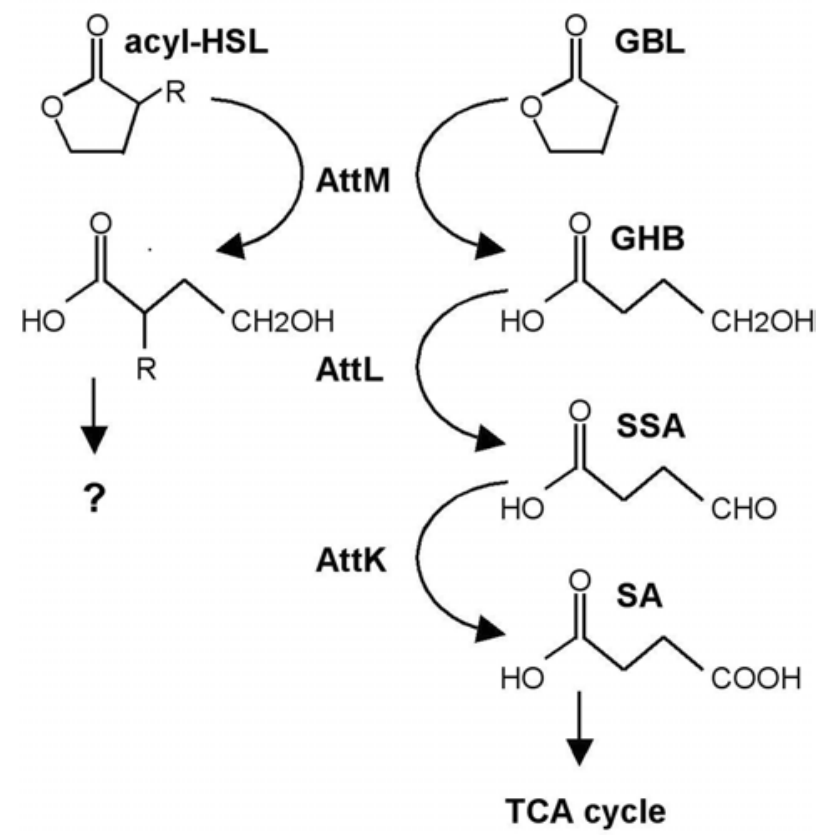

Fig. 2. Proposal for $\gamma$-butyrolactone (GBL)-assimilation pathway in Agrobacterium tumefaciens C58. The capacity of lactonase AttM to inactivate $\mathrm{N}$-acyl-homoserine lactone (acyl-HSL), such as $\mathrm{N}$-3-oxooctanoyl-L-homoserine lactone $\left(\mathrm{R}=\mathrm{NH}-\mathrm{CO}-\mathrm{CH}_{2}-\mathrm{CO}-\left(\mathrm{CH}_{2}\right)_{4}-\mathrm{CH}_{3}\right)$ and C6HSL ( $\left.\mathrm{R}=\mathrm{NH}-\mathrm{CO}-\left(\mathrm{CH}_{2}\right)_{4}-\mathrm{CH}_{3}\right)$, was described previously (Carlier et al. 2003; Zhang et al. 2002). The role of lactonase AttM, $\gamma$-hydroxybutyrate (GHB)-dehydrogenase AttL, and succinate semialdehyde (SSA)dehydrogenase AttK in GBL-assimilative pathway of A. tumefaciens C58 is discussed in this work. 
tion in A. tumefaciens C58 cultures. In contrast, when this bacterium grew in AB-GBL medium (data not shown) and in AB-mannitol medium, which was supplemented with the $a t t K$ inducers SSA, GHB, or GBL, it did not accumulate oxoC8HSL, whereas it did when grown in AB-mannitol supplemented with SA (Fig. 6). Moreover, A. tumefaciens C58 cells, in which the attKLM operon was induced by GBL, became able to inactivate exogenous C6HSL and oxo-C8HSL signals (Table 2). This acyl-HSL inactivating activity also was observed when the A. tumefaciens C58 cells were induced by GHB and SSA, whereas incubation in the presence of SA had no effect (Table 2). In similar experiments, the mutant $\mathrm{C} 101$ appeared to be insensitive to the addition of inducers, confirming the genetic link between the disappearance of acyl-HSL in A. tumefaciens C58 cultures and the expression of the GBL-degradation pathway encoded by attJKLM genes.
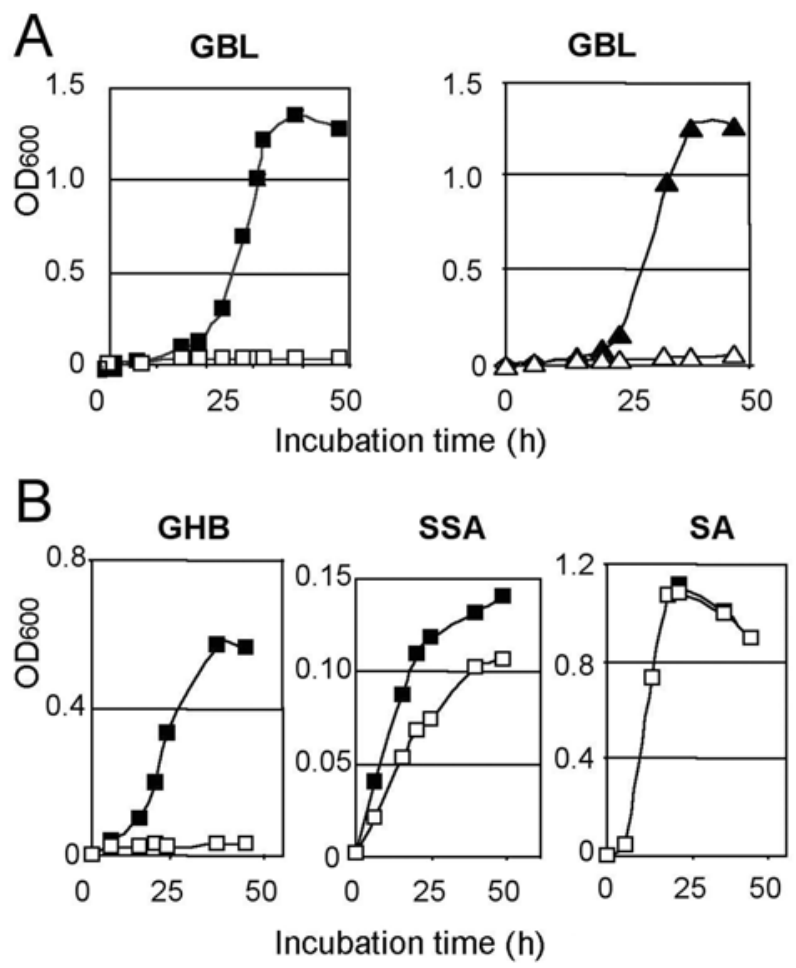

Fig. 3. Growth of Agrobacterium tumefaciens on $\gamma$-butyrolactone (GBL), $\gamma$-hydroxybutyrate (GHB), succinic acid (SA), and succinate semialdehyde (SSA). A, Growth of A. tumefaciens C58 (closed squares), A. tumefaciens C101 (open squares), and A. tumefaciens C58 harboring p6000 (closed triangles) or pMIR113 (open triangles), was monitored in ABGBL medium. B, Growth curves of A. tumefaciens C58 (closed squares) and C101 (open squares) in AB-GHB, AB-SSA, and AB-SA were compared. The experiments were done in triplicate; standard deviations were lower than symbol size.

\section{DISCUSSION}

A. tumefaciens C58 communicates using oxo-C8HSL and contains two lactonase-encoding genes, attM and aiiB (Carlier et al. 2003). In A. tumefaciens A6, the attKLM operon seems to be implied in the turnover of oxo-C8HSL (Zhang et al. 2002). In this article, a novel function of the attKLM operon is defined in A. tumefaciens C58.

Genetic analysis of the attJKLM genes supports their implication in an assimilative pathway for GBL and GHB. Aside from the demonstration that GBL assimilation interferes with QS regulation, this work constitutes the first description of a complete catabolic pathway of GBL in a bacterium (Fig. 2). Comparison between this bacterial pathway and that known in animals (Mason and Kerns 2003) evidences three common enzymatic steps: the opening of the GBL ring to yield GHB, the oxidation of GHB into SSA, and the oxidation of SSA into SA, a compound of the TCA cycle.
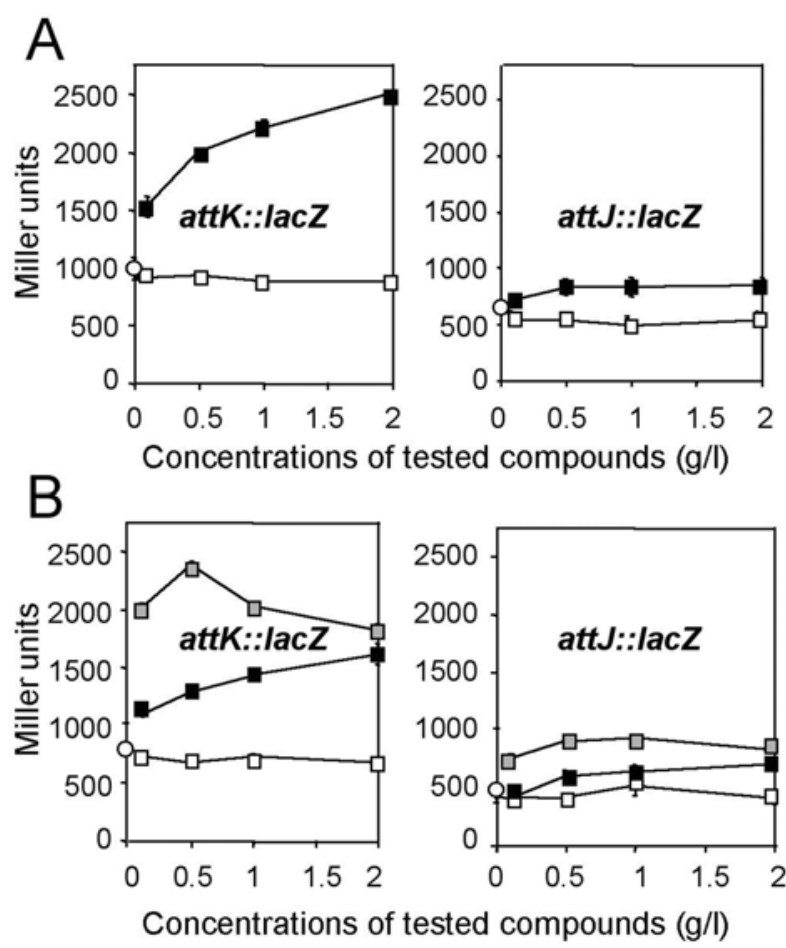

Fig. 4. Expression of the attJ::lacZ and $a t t K:: l a c Z$ transcriptional fusions in Agrobacterium tumefaciens C58. The $\beta$-galactosidase activity of the bacteria was measured from four independent cultures and expressed in Miller units. Prior to $\beta$-galactosidase assays, the cells were incubated for $2 \mathrm{~h}$ with $\mathbf{A}$, increasing concentrations of mannitol (open squares) and $\gamma$-butyrolactone (black squares) and $\mathbf{B}$, mannitol (open squares), $\gamma$ hydroxybutyrate (black squares), and succinate semialdehyde (gray squares). Open circles represent $\beta$-galactosidase activity after addition of water as control.

Table 1. Expression of attL and attM in Escherichia coli

\begin{tabular}{|c|c|c|c|c|c|c|}
\hline \multicolumn{2}{|c|}{ Plasmids harbored by E. coli DH5 $\alpha$} & \multicolumn{4}{|c|}{ Growth on different compounds as sole carbon source ${ }^{a}$} & \multirow[b]{2}{*}{ Inactivation $^{\mathrm{h}}$} \\
\hline Name & Expressed genes & SA & SSA & GHB & GBL & \\
\hline None & None & $1.07 \pm 0.02$ & $0.19 \pm 0.01$ & $\ldots$ & $\ldots$ & No \\
\hline pGEM & None & $0.85 \pm 0.01$ & $0.24 \pm 0.01$ & & $\ldots$ & No \\
\hline pMIR117 & $a t t L$ & $0.90 \pm 0.02$ & $0.33 \pm 0.01$ & $0.88 \pm 0.01$ & $\ldots$ & No \\
\hline pMIR102 & $a t t M$ & $0.84 \pm 0.02$ & $0.27 \pm 0.01$ & 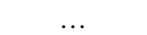 & $\ldots$ & Yes \\
\hline pMIR117 and p6010 & attL & $0.81 \pm 0.04$ & $0.30 \pm 0.05$ & $0.54 \pm 0.02$ & $\ldots$ & No \\
\hline pMIR117 and pMIR102 & attL, attM & $0.83 \pm 0.02$ & $0.23 \pm 0.01$ & $0.46 \pm 0.01$ & $0.82 \pm 0.14$ & Yes \\
\hline
\end{tabular}

${ }^{a}$ Optical density at $600 \mathrm{~nm}$ was measured daily during 3 days, only the final mean values were shown. SA $=$ succinic acid, SSA = succinate semialdehyde, $\mathrm{GHB}=\gamma$-hydroxybutyrate, $\mathrm{GBL}=\gamma$-butyrolactone.

${ }^{\mathrm{b}}$ Inactivation of $N$-acyl-homoserine lactone (acyl-HSL) signal measured after $24 \mathrm{~h}$ of incubation in presence of C6HSL at $25 \mu \mathrm{M} ; \ldots=$ no growth. 
In A. tumefaciens, these enzymatic reactions are likely to be catalyzed by AttM ( $\gamma$-butyrolactonase), AttL (homolog to alcohol dehydrogenases IV of COG1454), and AttK (homolog to NAD-dependent dehydrogenases of COG1012), respectively. The COG families were defined by Tatusov and associates (2001). Unlike SA and SSA, which are common intermediates in several bacterial and eukaryotic pathways, the occurrence of GHB and GBL in nature is quite limited. GHB essentially is described as the immediate catabolic intermediate of GBL, and as a psychoactive substance (Mason and Kerns 2003). On the other hand, GBL is produced for industrial purposes, and is found in plants (Lee and Shibamoto 2000) and in fermented beverages such as wines (Vose et al. 2000). The fact that $A$. tumefaciens evolved a GBL-catabolic operon strongly suggests that it may be confronted by this compound during its life in soils and on plant surface. In agreement with this hypothesis, proteome analysis showed that the proteins AttM and AttK were expressed by A. tumefaciens $\mathrm{C} 58$ when it was exposed to tomato roots (Rosen et al. 2003). Indeed, the catabolic property conferred by the attKLM genes could confer a selective advantage upon $A$. $t u$ mefaciens populations in such plant environments. However, this assertion needs to be investigated further.

In relation with the above problem, costs and benefits of the expression of attKLM should be examined, especially because this operon encodes the lactonase AttM that inactivates the acyl-HSL signals (Fig. 2). When the attKLM operon was not induced (i.e., in the absence of GBL, GHB, or SSA), the concentrations of oxo-C8HSL were identical in the culture supernatants of the wild-type strain C58 and its attJKLM mutant. Moreover, the acyl-HSLs, even added in excess to the growth media, do not induce the expression of the attKLM operon. All these features indicate that this operon would not participate, under these experimental conditions (in the absence of attKLM-inducers and traR-inducers), in the modulation of acyl-HSL level in A. tumefaciens C58. In A. tumefaciens A6, it was reported that the same operon may be implied in the turnover of oxo-C8HSL because its expression was induced when cells reached stationary phase (Zhang et al. 2002). In the case of A. tumefaciens C58, such growthphase-dependent regulation of the attKLM operon remains to be carefully analyzed before any premature comparison with A. tumefaciens A6. However, it should be underlined that $A$. tumefaciens $\mathrm{C} 58$ contains a second lactonase-encoding gene, aiiB (Carlier et al. 2003), that also might contribute to the modulation of the oxo-C8HSL signal.

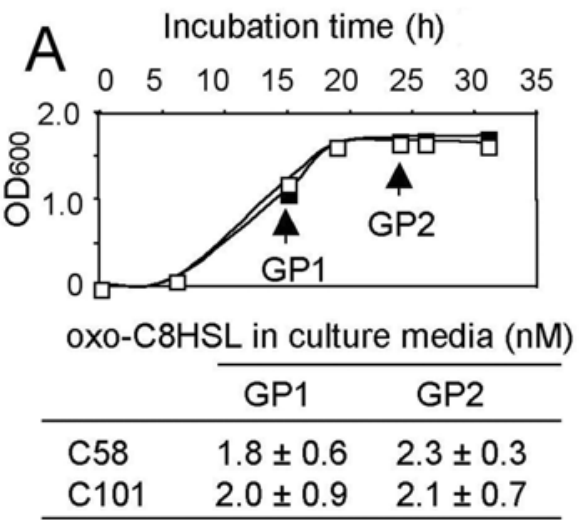

In the presence of GBL, GHB, or SSA, A. tumefaciens C58 clearly inactivates endogenous and exogenous acyl-HSL signals, because the expression of the attKLM operon and, more precisely, the expression of the lactonase AttM is induced. The large spectrum of acyl-HSL cleaved by this enzyme (Carlier et al. 2003; Zhang et al. 2002) suggests that A. tumefaciens C58, under attKLM induction conditions, is able to

SA $\square$ SSA $\square$ GHB $\square$ GBL
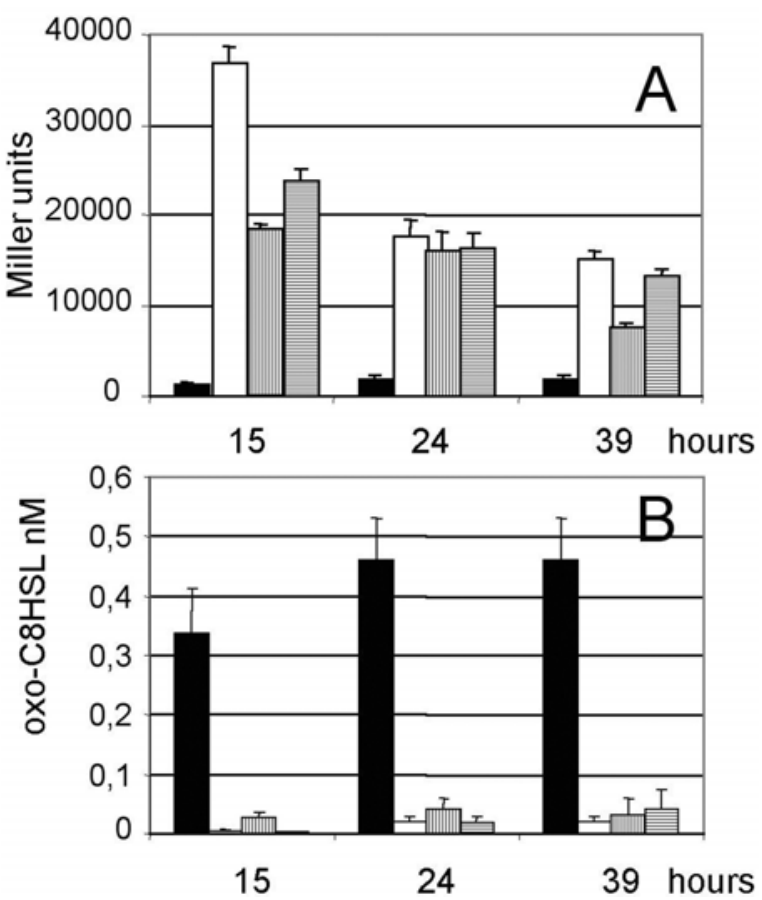

Fig. 6. $N$-acyl-homoserine lactone (acyl-HSL) level in the presence of attKLM-inducers. In all these experiments, the Agrobacterium tumefaciens strains were grown in AB-mannitol, supplemented with succinic acid (SA), succinate semialdehyde (SSA), $\gamma$-hydroxybutyrate (GHB), and $\gamma$-butyrolactone $(\mathrm{GBL})$ at $0.2 \mathrm{~g} / \mathrm{liter}$. When cells reached stationary phase, after 15,24 , and $39 \mathrm{~h}$ of culture, A, the expression of the attK::lacZ fusion was measured in A. tumefaciens C58 harboring pMIR122, whereas $\mathbf{B}$, the concentration of $\mathrm{N}$-3-oxo-octanoyl-L-homoserine lactone (oxo-C8HSL) was evaluated in A. tumefaciens C58 culture medium. In the presence of SSA, GHB, and GBL, the attK::lacZ fusion was highly expressed, whereas a low concentration of oxo-C8HSL was detected in culture supernatants of A. tumefaciens C58.

B

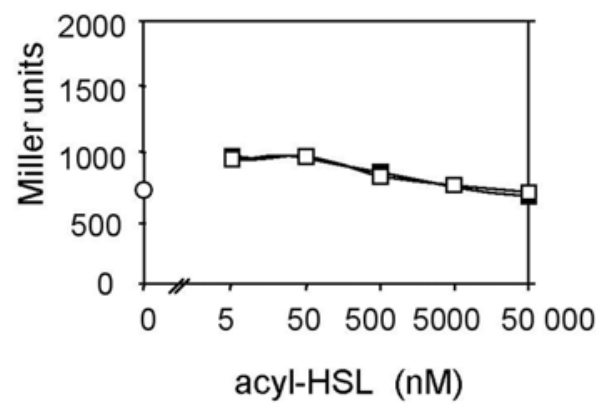

Fig. 5. $N$-acyl-homoserine lactone (acyl-HSL) accumulation in the absence of attKLM-inducers. In all these experiments, the Agrobacterium tumefaciens strains were cultivated in AB-mannitol. A, Concentration of $\mathrm{N}$-3-oxo-octanoyl-L-homoserine lactone (oxo-C8HSL) in culture medium was compared during the growth of $A$. tumefaciens $\mathrm{C} 58$ (closed squares) and $\mathrm{C} 101$ (open squares). B, Expression of the attK::lacZ fusion was measured in A. tumefaciens C58, in the presence of increasing concentrations of C6HSL (open squares) and oxo-C8HSL (closed squares). Open circle represents $\beta$-galactosidase activity after addition of water as control. 
disrupt any acyl-HSL signal; therefore, it becomes an efficient acyl-HSL-degrading bacterium. The attM-paralogous gene $a i i B$, that also encodes lactonase activity directed toward acyl-HSLs (Carlier et al. 2003), likely does not participate in this inducible acyl-HSL-degrading activity, because the attJKLM mutant that still harbors the $a i i B$ gene on its pTi was unable to degrade acyl-HSL even in the presence of attKLM inducers. However, the physiological conditions in which the $a i i B$ gene is expressed remain to be clarified before any role can be attributed to this gene and to the encoded peptide.

A few functions only are known to be determined by At plasmid genes (Mathysse and McMahan 2001; Nair et al. 2003; Vaudequin-Dransart et al. 1998), whereas the pTi harbors most of the genes required for plant tumorigenesis ( $\mathrm{Zhu}$ et al. 2000), as well as those involved in the synthesis (traI) and sensing (traR) of the QS signal, oxo-C8HSL. At this stage of knowledge about QS regulation in A. tumefaciens C58, the Ti plasmid may be regarded as an acyl-HSL emitter, and the At plasmid as an inducible acyl-HSL disrupter. Such a distribution of functions on separate replicons, but within the same bacterial cell, should be considered carefully with respect to further ecological interpretation and integration in the complex QS network of A. tumefaciens (Piper et al. 1993; Qin et al. 2000; Swiderska et al. 2001; Zhang et al. 1993; Zhu and Winans 2001). Similarly, novel acyl-HSL hydrolases (i.e., the $N$-acylases belonging to the family AiiD) have been described in different bacteria, such as Ralstonia sp. strain $\mathrm{XJ} 12 \mathrm{~B}$ and Pseudomonas aeruginosa PAO1 (Huang et al. 2003; Lin et al. 2003). The determination of the physiological conditions in which these acyl-HSL-degrading enzymes are expressed will constitute valuable and necessary data to assign biological functions to these enzymes, as in the case of the AiiA-lactonases related to AttM (Dong et al. 2002; Park et al. 2003).

\section{MATERIALS AND METHODS}

\section{Bacterial strains.}

A. tumefaciens C58, as well as its derivatives C58.C1, C58.C2, and C58.00 (Vaudequin-Dransart et al. 1998), which are cured for $\mathrm{pTi}, \mathrm{pAt}$, and, $\mathrm{pTi}$ and $\mathrm{pAt}$, respectively, were used in this study. The $\Delta($ attJKLM)::aph mutant (Fig. 1), named A. tumefaciens C101, was obtained from strain C58 by a standard procedure for gene replacement via the introduction of a suicide plasmid by electroporation (Ugalde et al. 1998). Briefly, the aph gene purified from the plasmid p34S$\mathrm{Km}$ (Dennis and Zyltra 1998) was used to replace the attJKLM region between nucleotides 143,303 and 146,511 (gi:17938588), according to the pAtC58 sequence (Wood et al. 2001). A. tumefaciens NTLR4 (Cha et al. 1998) and Chromobacterium violaceum CV026 (McClean et al. 1997) were used as acyl-HSL biosensors for oxo-C8HSL and C6HSL, respectively. E. coli $\mathrm{DH} 5 \alpha$ was the routine host for cloning and gene expression experiments.

\section{Plasmids.}

The maps of plasmids used in this study are given in Figure 1. The plasmid pMIR102, that confers the constitutive expression of the lactonase-encoding gene attM upon its host, was described previously (Carlier et al. 2003). The attL DNA region was amplified using primers 5'-ATACCTGTGCTCGGCCATC and 5'-TGCTGTCAGAAATGGGTCAG, and cloned into the pGEM-T easy cloning vector (Promega Corp., Madison, WI, U.S.A.) to yield pMIR117. Plasmid pMIR113 is a derivative of the broad host-range plasmid pME6000 (Maurhofer et al. 1998), in which an amplified fragment overlapping the attJ gene (primers 5'-CAAACCATCGACGCAATATG and 5'-GCGGGATCCCTGGGGTATTGG) was cloned at the BamHI and PstI sites. The divergent promoters of attJ and attKLM were amplified as a single DNA fragment (primers 5'-TCAGCCATGCACTATCCTTGA and 5'-GTCTAGCCATCCGCGGCTT) and fused with the promoterless lacZ-aph cassette of pKOK5 (Kokotek and Lotz 1989). Then, the attJ::lacZ and attK::lacZ transcriptional fusions were subcloned as $S p h I-S a c I$ fragments into the broad host-range vector pME6031 (Heeb et al. 2000) to yield plasmids pMIR121 and pMIR122, respectively.

\section{Growth conditions.}

E. coli was cultivated at $37^{\circ} \mathrm{C}$ in Luria Bertani rich medium and in M9 minimal medium (Sambrook et al. 1989), supplemented with NAD (0.66 g/liter) and the required carbon source ( $2 \mathrm{~g} / \mathrm{liter})$. A. tumefaciens strains were cultivated at $30^{\circ} \mathrm{C}$ in tryptone yeast medium and in $\mathrm{AB}$ minimal medium (Chilton et al. 1974), supplemented with $\mathrm{NH}_{4} \mathrm{Cl}$ (1g/liter) and one of the following carbon sources ( $2 \mathrm{~g} / \mathrm{liter})$ : mannitol, GBL, $\gamma$-valerolactone, homoserine lactone, GHB, or SA. In growth experiments, SSA was used at $0.3 \mathrm{~g} /$ liter. Mannitol was used as carbon source in all Agrobacterium precultures. The growth curves of $A$. tumefaciens and $E$. coli were computed by monitoring the optical density at $600 \mathrm{~nm}$. When appropriate, antibiotics were used at the following concentrations: ampicillin at $50 \mathrm{mg} /$ liter, kanamycin at $50 \mathrm{mg} / \mathrm{liter}$, and tetracycline at $10 \mathrm{mg} / \mathrm{liter}$.

\section{Expression of transcriptional fusion.}

The $\beta$-galactosidase activity was measured with $o$-nitrophenyl- $\beta$-D-galactopyranoside as a substrate, and expressed in Miller units (Sambrook et al. 1989). Four independent cultures were used for each experimental condition. In induction experiments, the cells containing the transcriptional fusions were cultivated in AB-mannitol medium for $24 \mathrm{~h}$. Then, $360 \mu \mathrm{l}$ of these cultures were mixed with $40 \mu \mathrm{l}$ of the solutions of the compound to assay and incubated for $2 \mathrm{~h}$ at $24^{\circ} \mathrm{C}$ until the $\beta$ galactosidase assay was performed.

\section{Acyl-HSL quantification.}

The acyl-HSLs were quantified on KC18 reversed thin-layer chromatography (TLC) plates (Whatman, Maidstone, England) by comparison with an appropriate range of standards, as previously described (Cha et al. 1998; McClean et al. 1997). The

Table 2. Inactivation of exogenous $N$-acyl-homoserine lactones (acyl-HSLs) by Agrobacterium tumefaciens $\mathrm{C} 58^{\mathrm{a}}$

\begin{tabular}{|c|c|c|c|c|c|c|c|}
\hline \multirow[b]{2}{*}{ Bacterial strains } & \multicolumn{5}{|c|}{ Inactivation of C6HSL $(25 \mu \mathrm{M})$} & \multicolumn{2}{|c|}{$\begin{array}{c}\text { Inactivation of } \\
\text { oxo-octanoyl-L-homoserine lactone }(10 \mu \mathrm{M}) \\
\end{array}$} \\
\hline & Mannitol & GBL & GHB & SSA & SA & Mannitol & GBL \\
\hline A. tumefaciens C58 & No & Yes & Yes & Yes & No & No & Yes \\
\hline A. tumefaciens $\mathrm{C} 101$ & No & No & No & No & No & No & No \\
\hline
\end{tabular}

a Degradation assay were performed with washed cells of A. tumefaciens $\mathrm{C} 58$ and $\mathrm{C} 101$. Before degradation assay, cells were incubated in the presence of mannitol, $\gamma$-butyrolactone (GBL), $\gamma$-hydroxybutyrate (GHB), succinate semialdehyde (SSA), and succinic acid (SA). "No" indicates more than 80\% of the introduced acyl-HSLs remained after a 6-h incubation time, and "Yes" indicates less than 5\% of the introduced acyl-HSLs remained after a 1-h incubation time. 
oxo-C8HSL produced by $A$. tumefaciens were extracted with ethyl acetate from 1-ml or 8-ml samples of Agrobacteriumculture supernatants and concentrated 100 times before quantification. An acyl-HSL-disappearance assay was performed in E. coli after C6HSL supplementation $(25 \mu \mathrm{M})$ to the medium, as previously described (Carlier et al. 2003), and in A. tumefaciens after supplementation by C6HSL $(25 \mu \mathrm{M})$ and oxoC8SHL $(10 \mu \mathrm{M})$. In these experiments, stationary phase cultures of $A$. tumefaciens were centrifuged and resuspended to a cell density of $5 \times 10^{7} \mathrm{CFU} / \mathrm{ml}$ into fresh $\mathrm{AB}$ medium containing mannitol, GBL, GHB, SSA, or SA as sole carbon source. After a 15-h incubation under shaking, the induced cells were washed with $\mathrm{NaCl}(0.8 \%)$ and inoculated at $10^{9} \mathrm{CFU} / \mathrm{ml}$ into fresh $\mathrm{AB}$ medium supplemented with mannitol $(0.2 \mathrm{~g} / \mathrm{liter})$ and $\mathrm{NH}_{4} \mathrm{Cl}(0.1 \mathrm{~g} / \mathrm{liter})$, and with C6HSL $(25 \mu \mathrm{M})$ or oxoC8SHL $(10 \mu \mathrm{M})$. After $1,2,3$, and $6 \mathrm{~h}$ of incubation, the presence of residual acyl-HSLs in the culture media was assayed as described above. In all degradation assays, the complete disappearance of acyl-HSL was verified by separating samples in a methanol/water (60:40) solvent system used to develop KC18 reversed TLC plates.

\section{ACKNOWLEDGMENTS}

We thank P. Williams (Nottingham, U.K.) for kindly providing pure oxo-C8HSL. Funding for this work was provided by the French Government via the Centre National de la Recherche Scientifique. The authorization to hold and manipulate GHB was given by the Agence Française de Sécurité Sanitaire des Produits de Santé.

\section{LITERATURE CITED}

Camara, M., Williams, P., and Hardman, A. 2002. Controlling infection by tuning in and turning down the volume of bacterial small-talk. Lancet Infect. Dis. 2:667-676.

Carlier, A., Uroz, S., Smadja, B., Latour, X., Fray, R., Dessaux, Y., and Faure, D. 2003. The Ti plasmid of Agrobacterium tumefaciens harbors an $a t t M$ paralogous gene, aiiB, also encoding $N$-acyl homoserine lactonase activity. Appl. Environ. Microbiol. 69:4989-4993.

Cha, C., Gao, P., Chen, Y. C., Shaw, P. D., and Farrand, S. K. 1998. Production of acyl-homoserine lactone quorum-sensing signals by gramnegative plant-associated bacteria. Mol. Plant-Microbe Interact. 11:1119-1129.

Chilton, M. D., Currier, T. C., Farrand, S. K., Bendich, A. J., Gordon, M. P., and Nester, E. W. 1974. Agrobacterium tumefaciens and PS8 bacteriophage DNA not detected in crown gall tumor DNA. Proc. Natl. Acad. Sci. U.S.A. 71:3672-3676.

Dennis, J., and Zylstra, G. 1998. Plasposons: modular self-cloning minitransposon derivatives for rapid genetic analysis of gram-negative bacterial genomes. Appl. Environ. Microbiol. 64:2710-2715.

Dong, Y. H., Gusti, A. R., Zhang, Q., Xu, J. L., and Zhang, L. H. 2002. Identification of quorum-quenching $N$-acyl homoserine lactonases from Bacillus species. Appl. Environ. Microbiol. 68:1754-1759.

Dong, Y. H., Wang, L. H., Xu, J. L., Zhang, H. B., Zhang, X. F., and Zhang, L. H. 2001. Quenching quorum-sensing-dependent bacterial infection by an $N$-acyl homoserine lactonase. Nature 411:813-817.

Dong, Y. H., Xu, J. L., Li, X. Z., and Zhang, L. H. 2000. AiiA, an enzyme that inactivates the acylhomoserine lactone quorum-sensing signal and attenuates the virulence of Erwinia carotovora. Proc. Natl. Acad. Sci. 97:3526-3531.

Fuqua, C., and Greenberg, E. P. 2002. Listening in on bacteria: acyl-homoserine lactone signaling. Nature Rev. 3:685-695.

Fuqua, W. C., Winans, S. C., and Greenberg, E. P. 1994. Quorum sensing in bacteria: the LuxR-LuxI family of cell density-responsive transcriptional regulators. J. Bacteriol. 176:269-275.

Heeb, S., Itoh, Y., Nishijyo, T., Schnider, U., Keel, C., Wade, J., Walsh, U., O'Gara, F., and Haas, D. 2000. Small, stable shuttle vectors based on the minimal pVS1 replicon for use in gram-negative, plant-associated bacteria. Mol. Plant-Microbe Interact. 13:232-237.

Henzter, M., Wu, H., Andersen, J. B., Riedel, K., Rasmussen, T. B., Bagge, N., Kumar, N., Schembri, M. A., Song, Z., Kristoffersen, P., Manafield, M., Costerton, J. M., Molin, S., Eberl, L., Steinberg, P., Kjelleberg, S., Hoiby, N., and Givskov, M. 2003. Attenuation of Pseudomonas aeruginosa virulence by quorum sensing inhibitors. EMBO (Eur. Mol. Biol. Organ.) J. 22:3803-3815.
Hoang, T. T., and Schweizer, H. P. 1999. Characterization of Pseudomonas aeruginosa enoyl-acyl carrier protein reductase (FabI): a target for the antimicrobial triclosan and ist role in acylated homoserine lactone synthesis. J. Bacteriol. 191:5489-5497.

Huang, J. J., Han, J. I., Zhang, L. H., and Leadbetter, J. R. 2003. Utilization of acyl-homoserine lactone quorum signals for growth by a soil pseudomonad and Pseudomonas aeruginosa PAO1. Appl. Environ. Microbiol. 69:5941-5949.

Kokotek, W., and Lotz, W. 1989. Construction of a lacZ-kanamycin-resistance cassette, useful for site-directed mutagenesis and as a promoter probe. Gene 84:467-471.

Leadbetter, J. R., and Greenberg, E. P. 2000. Metabolism of acyl-homoserine lactone quorum-sensing signals by Variovorax paradoxus. J. Bacteriol. 182:6921-6926.

Lee, K. G., and Shibamoto, T. 2000. Antioxidant properties of aroma compounds isolated from soybeans and mung beans. J. Agric. Food Chem. 48:4290-4293.

Lin, Y. H., Xu, J. L., Hu, J., Wang, L. H., Ong, S. L., Leadbetter, J. R., and Zhang, L. H. 2003. Acyl-homoserine lactone acylase from Ralstonia strain XJ12B represents a novel and potent class of quorum-quenching enzymes. Mol. Microbiol. 47:849-860.

Manefield, M., Rasmussen, T. B., Henzter, M., Andersen, J. B., Steinberg, P., Kjelleberg, S., and Givskov, M. 2002. Halogenated furanones inhibit quorum sensing through accelerated LuxR turnover. Microbiology 148:1119-1127.

Mason, P. E., and Kerns, W. P., II. 2003. Gamma hydrobutyric acid (GHB) intoxication. Acad. Emerg. Med. 9:730-739.

Mathysse, A. G., and McMahan, S. 2001. The effect of the Agrobacterium tumefaciens attR mutation on attachment and root colonization differs between legumes and other dicots. Appl. Environ. Microbiol. 67:10701075 .

Maurhofer, M., Reimann, C., Schimidli-Sacherer, P., Heeb, S., Haas, D., and Défago, G. 1998. Salicylic acid biosynthesis genes expressed in Pseudomonas fluorescens strain P3 improve the induction of systemic resistance in tobacco against tobacco necrosis virus. Phytopathology 88:678-684.

McClean, K. H., Winson, M. K., Fish, L., Taylor, A., Chhabra, S. R., Camara, M., Daykin, M., Lamb, J. H., Swift, S., Bycroft, B. W., Stewart, G. S., and Williams, P. 1997. Quorum-sensing and Chromobacterium violaceum: exploitation of violacein production and inhibition for the detection of N-acylhomoserine lactones. Microbiology 143:3703-3711.

Nair, G. R., Liu, Z., and Binns, A. N. 2003. Reexamining the role of the accessory plasmid pAtC58 in the virulence of Agrobacterium tumefaciens strain C58. Plant Physiol. 133:989-999.

Park, S. Y., Lee, S. J., Oh, T. K., Oh, J. W., Koo, B. T., Yum, D. Y., and Lee, J. K. 2003. AhlD, an $N$-acylhomoserine lactonase in Arthrobacter sp., and predicted homologues in other bacteria. Microbiology 149:1541-1550.

Piper, K. R., Beck von Bodman, S., and Farrand, S. K. 1993. Conjugation factor of Agrobacterium tumefaciens regulates Ti plasmid transfer by autoinduction. Nature 362:448-450.

Qin, Y., Luo Z. Q., Smyth, A. J., Gao, P., Beck van Bodman, S., and Farrand, S. K. 2000. Quorum-sensing signal binding results in dimerization of TraR and its release from membranes into the cytoplasm EMBO (Eur. Mol. Biol. Organ.) J. 19:5212-5221.

Rosen, R., Matthysse, A. G., Becher, D., Biran, D., Yura, T., Hecker, M., and Ron, E. Z. 2003. Proteome analysis of plant-induced proteins of Agrobacterium tumefaciens. FEMS (Fed. Eur. Microbiol. Soc.) Microbiol. Lett. 44:355-360.

Sambrook, J., Fritsch, E. F., and Maniatis, T. 1989. Molecular Cloning: A Laboratory Manual, 2nd ed. Cold Spring Harbor Laboratory Press, Cold Spring Harbor, NY, U.S.A.

Swiderska, A., Berndtson, A. K., Cha, M. R., Li, L., Beaudouin, G. M. J., Zhu, J., and Fuqua, C. 2001. Inhibition of the Agrobacterium tumefaciens TraR quorum-sensing regulator. Interactions with the TraM antiactivator. J. Biol. Chem. 276:49449-49458.

Tatusov R. L., Natale, D. A., Garkavtsev, I. V., Tatusova, T. A., Shankavaram, U. T., Rao, B. S., Kiryutin, B., Galperin, M. Y., Fedorova, N. D., and Koonin, E. V. 2001. The COG database: new developments in phylogenetic classification of proteins from complete genomes. Nucleic Acids Res. 29:22-28.

Ugalde, J. E., Lepek, V., Uttario, A., Estrella, J., Iglesias, A., and Ugalde, R. A. 1998. Gene organization and transcription analysis of the Agrobacterium tumefaciens glycogen ( $\mathrm{glg}$ ) operon: two transcripts for the single phosphoglucomutase gene. J. Bacteriol. 180:6557-6564.

Uroz, S., D’Angelo-Picard, C., Carlier, A., Elasri, M., Sicot, C., Petit, A., Oger, P., Faure, D., and Dessaux, Y. 2003. Novel bacteria degrading $N$ acyl homoserine lactones and their use as quenchers of quorum-sensing regulated functions of plant pathogenic bacteria. Microbiology 149:1981-1989. 
Vaudequin-Dransart, V., Petit, A., Chilton, W. S., and Dessaux, Y. 1998. The cryptic plasmid of Agrobacterium tumefaciens cointegrates with the $\mathrm{Ti}$ plasmid and cooperates for opine degradation. Mol. PlantMicrobe Interact. 11:583-591.

Vose, J., Tighe, T., Schwartz, M., and Buel, E. 2000. Detection of gammabutyrolactone (GBL) as a natural component in wine. J. Forensic Sci. 46:1164-1167.

Whitehead, N. A., Barnard, A. M. L., Slater, H., Simpson, N. J. L., and Salmond, G. P. C. 2001. Quorum sensing in Gram-negative bacteria FEMS (Fed. Eur. Microbiol. Soc.) Microbiol. Rev. 25:365-404.

Wood, D. W., Setubal, J. C., Kaul, R., Monks, D. E., Kitajima, J. P., Okura, V. K., Zhou, Y., Chen, L., Wood, G. E., Almeida, N. F. Jr, Woo, L., Chen, Y., Paulsen, I. T., Eisen, J. A., Karp, P. D., Bovee, D. Sr., Chapman, P., Clendenning, J., Deatherage, G., Gillet, W., Grant, C., Kutyavin, T., Levy, R., Li, M. J., McClelland, E., Palmieri, A., Raymond, C., Rouse, G., Saenphimmachak, C., Wu, Z., Romero, P., Gordon, D., Zhang, S., Yoo, H., Tao, Y., Biddle, P., Jung, M., Krespan, W., Perry, M., Gordon-Kamm, B., Liao, L., Kim, S., Hendrick, C.,
Zhao, Z. Y., Dolan, M., Chumley, F., Tingey, S. V., Tomb, J. F., Gordon, M. P., Olson, M. V., and Nester, E. W. 2001. The genome of the natural genetic engineer Agrobacterium tumefaciens C58. Science, 294:23172323.

Zhang, H. B., Wang, L. H., and Zhang, L. H. 2002. Genetic control of quorum-sensing signal turnover in Agrobacterium tumefaciens. Proc. Natl. Acad. Sci. U.S.A. 99:4638-4643.

Zhang, L., Murphy, P. J., Kerr, A., and Tate, M. E. 1993. Agrobacterium conjugation and gene regulation by $\mathrm{N}$-acyl-L-homoserine lactones. Nature 362:446-448.

Zhang, L. H. 2002. Quorum quenching and proactive host defense. Trends Plant Sci. 8:238-244.

Zhu, J., Oger, P. M., Schrammeijer, B., Hooykaas, P. J. J., Farrand, S. K. and Winans, S. C. 2000. The bases of Crown Gall tumorigensis. J. Bacteriol. 14:3885-3895.

Zhu, J., and Winans, C. 2001. The quorum-sensing transcriptional regulator TraR requires its cognate signaling ligand for protein folding, protease resistance, and dimerization. Proc. Natl. Acad. Sci. U.S.A. 98:1507-1512. 